\title{
Navigating Intermediate Targets: The Nervous System Midline
}

\author{
Barry J. Dickson ${ }^{1}$ and Yimin Zou ${ }^{2}$ \\ ${ }^{1}$ Research Institute of Molecular Pathology (IMP), Dr. Bohrgasse 7, A-1030 Vienna, Austria \\ ${ }^{2}$ Division of Biological Sciences, Section of Neurobiology, University of California, San Diego, \\ La Jolla, California 92093-0366 \\ Correspondence: dickson@imp.ac.at and yzou@ucsd.edu
}

In a bilaterally symmetric animal, the midline plays a key role in directing axon growth during wiring of the nervous system. Midline cells provide a variety of guidance cues for growing axons, to which different types of axons respond in different ways and at different times. For some axons, the midline is an intermediate target. They first seek it out, but then move on towards their final targets on the opposite side. For others, the midline is a repulsive barrier that keeps them on their own side of the midline. And for many of these axons the midline provides signals that guide them along specific lateral pathways or up and down the longitudinal axis.

$T^{\mathrm{h}}$ he complex guidance decisions at the midline have made it a particularly fascinating model for investigating the mechanisms and logic of axon pathfinding. Much of this work has focused on the ventral midline of the vertebrate spinal cord and its Drosophila analog, the ventral nerve cord. This work has sought to explain why some axons cross the midline, whereas others do not; why these axons respond differently to midline cues before and after crossing; and how the midline directs axon traffic along the lateral pathways. Striking similarities, as well as intriguing differences, have been documented in the way these guidance decisions are regulated in vertebrates and in Drosophila. Here, we briefly introduce the two systems, then review our current understanding of each of the key guidance decisions, and finally discuss some of the general principles and open questions that have emerged from these studies.

\section{AXON GUIDANCE AT THE CNS MIDLINE}

At the ventral midline of the developing vertebrate spinal cord is a transient structure composed of ependymal cells called the floor plate. The floor plate plays a critical role in patterning neuronal fates and projections within the spinal cord. It is a source of morphogens that specify neuronal cell fates along the dorsal-ventral axis, as well as guidance molecules that orient the growth of their axons. In flies, the midline is instead composed of both neurons and glia, most of which persist through development to form an integral part of the larval central

Editors: Marc Tessier-Lavigne and Alex L. Kolodkin

Additional Perspectives on Neuronal Guidance available at www.cshperspectives.org

Copyright (C) 2010 Cold Spring Harbor Laboratory Press; all rights reserved; doi: 10.1101/cshperspect.a002055

Cite this article as Cold Spring Harb Perspect Biol 2010;2:a002055 
nervous system. The midline of the fly embryo is not a major source of developmental morphogens, but it does share the floor plate's function in axon guidance. In both vertebrates and insects, the midline sculpts an orthogonal array of axonal projections. Commissural axons extend across the midline, forming the critical conduit for information flow between the left and right sides of the body. Longitudinal tracts run parallel to the midline, and include the axons that carry ascending and descending signals to and from the brain.

In vertebrates, the commissural neurons that are commonly used for axon guidance studies are born in the dorsal spinal cord and extend axons ventrally toward the floor plate during embryonic day 10 (E10) to E13.5. They first grow along a circumferential route, and then turn medially toward the floor plate before crossing under it to emerge on the contralateral side. From his detailed histological analyses of these projections (Fig. 1A-C), Cajal correctly predicted that the floor plate-the neuroepithelial wedge-might secrete a chemoattractant for the ventral orientation of commissural axons, and that these axons might respond to distinct guidance cues before and after entering and crossing the midline (Ramón y Cajal 1995).

In the fly nerve cord, commissural axons typically grow on a direct medial path from their lateral origin to the midline, crossing in either of the two main commissures in each segment. Whereas commissural neurons are segregated in the dorsal half of the spinal cord, in the fly nerve cord they are intermingled with ipsilateral interneurons and motor neurons. A particular advantage of the Drosophila system is the stereotypy of these projections and the availability of a range of genetic markers, which together allow the projections of specific, identifiable neurons to be studied in various genetic backgrounds and at cellular resolution (Fig. 1D-F).

Once they have crossed the midline, most vertebrate and many fly commissural axons turn anteriorly or posteriorly into a longitudinal pathway. They are joined in these longitudinal pathways by a smaller population of ipsilateral neurons. Although they project right alongside the midline, ipsilateral longitudinal axons do not cross it. Commissural axons of the vertebrate spinal cord and those at the fly midline therefore cross only once, and ipsilateral axons never.

Efforts to understand how midline cells direct the formation of these orthogonal axonal arrays have centered on five key questions. First, what guides commissural axons towards the midline? Second, why do commissural axons but not longitudinal axons cross the midline? Third, what changes as commissural axons cross the midline, causing them to behave differently before and after crossing? Fourth, how do axons, both commissural and longitudinal, choose between anterior and posterior pathways? And fifth, how are longitudinal axons sorted into discrete fascicles, each a characteristic distance from the midline? We examine each of these questions in turn, for both the vertebrate and invertebrate systems.

\section{GUIDING COMMISSURAL AXONS TOWARD THE MIDLINE}

One of the first steps in wiring up the fly ventral nerve cord and the vertebrate spinal cord is the directed growth of commissural axons toward the midline. In Drosophila, the cell bodies of commissural neurons are mostly located within a few tens of microns from the midline. This has made it somewhat difficult to study the initial growth of commissural axons across this short distance in any detail. In vertebrates, in contrast, the commissural neurons are located in the dorsal spinal cord, up to several hundred microns away from the floor plate. This has enabled the development of a variety of explant assays, which have been exploited in combination with genetic and biochemical studies to define the factors that guide the initial growth of commissural axons.

Using such explant assays, the floor plate was first found to secrete factors that promote both the outgrowth and attractive turning of commissural axons (Tessier-Lavigne et al. 1988). This assay served as the basis for a biochemical purification of an outgrowth-promoting factor, Netrin-1, which turned out to induce turning as well (Serafini et al. 1994; Kennedy et al. 1994). 
A

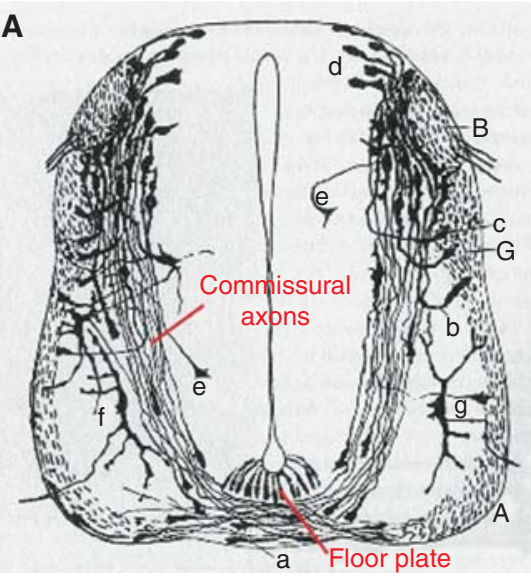

D

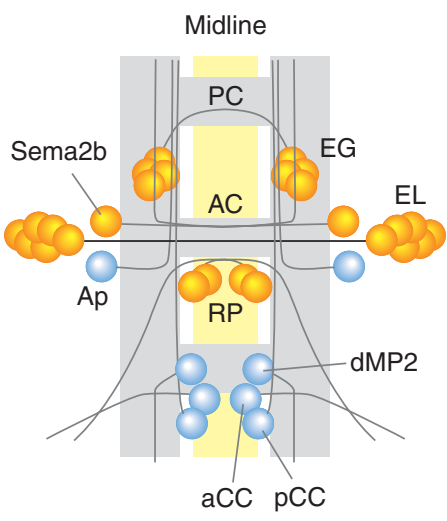

Navigating Intermediate Targets: The Nervous System Midline
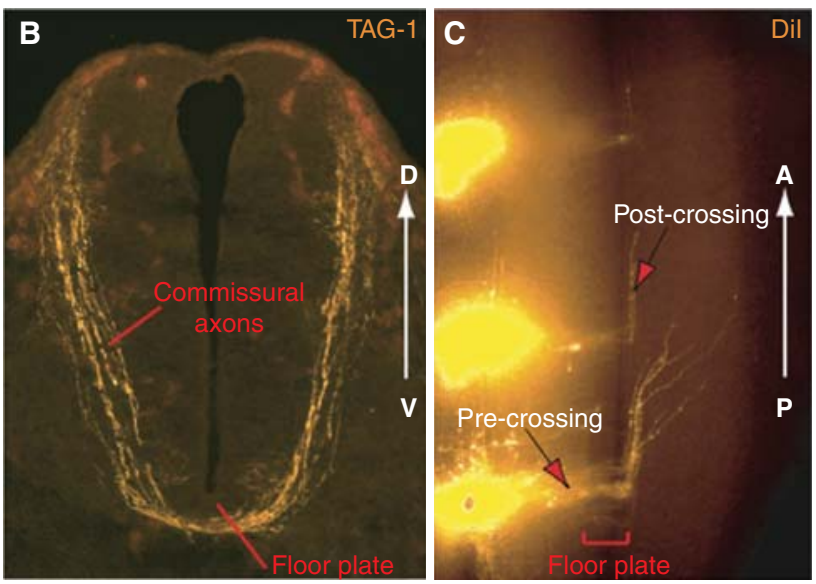

E

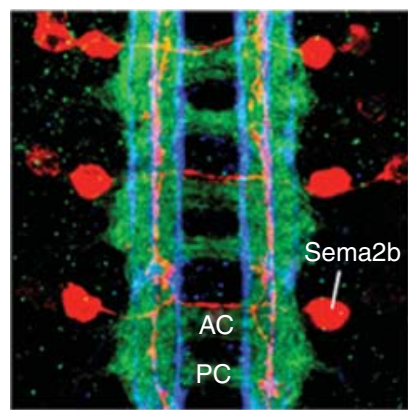

$\mathbf{F}$

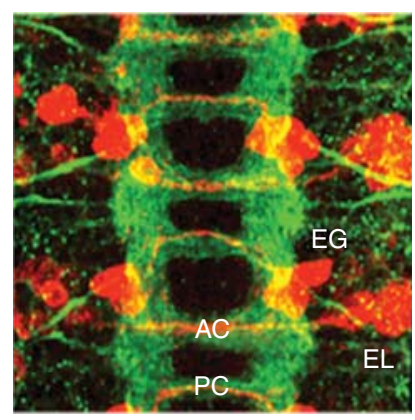

Figure 1. Commissural axons at the midline in vertebrates and Drosophila. (A) Drawing by Cajal showing commissural axons by Golgi staining of E4 chick embryonic spinal cord. Note the growth cones at the tip of axons. (B) Commissural axons revealed by immunostaining with a marker, TAG-1, in an E11.5 mouse spinal cord transverse section (similar stage as E4 chick embryo). (C) Midline crossing and anterior turning of commissural axons revealed by DiI tracing in E11.5 mouse open-book spinal cord preparation. $(D)$ Schematic of the ventral nerve cord of a late stage Drosophila embryo, anterior up. Yellow indicates midline cells, gray indicates axon tracts (adapted from Keleman et al. 2002). AC, anterior commissure; PC, posterior commissure. Examples of identified neurons and their projections are shown, with commissural neurons in orange and ipsilateral neurons in blue. Sema2b, an intersegmental commissural neuron; EG and EL, intrasegmental commissural neurons; RP, commissural motorneurons; dMP2, ipsilateral intersegmental neuron with posterior projections; pCC, ipsilateral intersegmental neuron with anterior projection; $\mathrm{aCC}$, ipsilateral motorneuron. (E) Confocal image of the nerve cord, with all axons stained in green, specific longitudinal pathways in blue (anti-FasII), and Sema2b neurons in red (adapted from Rajagopalan et al. 2000b). ( F ) Confocal image of the nerve cord with all axons stained in green and EG and EL neurons in red (adapted from Brankatschk and Dickson 2006).

Netrin-1 is expressed in a dorsal-to-ventral graded fashion in the spinal cord (Kennedy et al. 2006), and is required for the ventral growth of commissural axons in vivo (Serafini et al. 1996) (Fig. 2). The attractive response to Netrins is mediated by a transmembrane receptor, DCC (deleted in colorectal cancer) (Keino-Masu et al. 1996). Another putative Netrin receptor, DsCAM, may act in parallel to DCC to mediate the growth of commissural axons into and through the midline (Ly et al. 2008; Liu et al. 2009). 


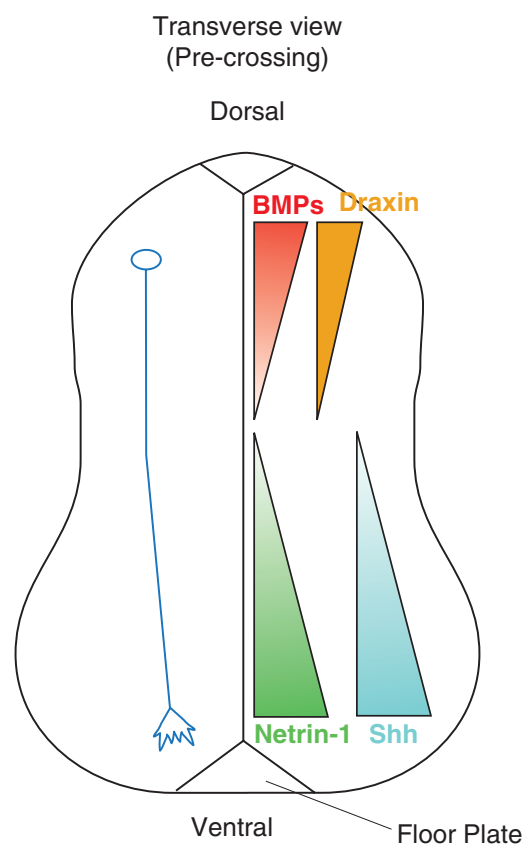

Figure 2. Multiple guidance cues control the pathfinding of spinal cord commissural axons along the dorsal-ventral axis. In a transverse view, precrossing commissural axons (blue axon) are attracted by Netrin-1 (green) and Shh (cyan), which are highly enriched in the floor plate and present in the ventral to dorsal decreasing gradient. Chemorepellants, such as the BMPs (red) and Draxin (orange), repel commissural axons from the dorsal midline to orient commissural axons to grow ventrally.

In netrin- 1 mutant mice, some commissural axons still grow into the floor plate. Moreover, the floor plate from netrin-1 mutant mice is still able to induce turning of commissural axons in explant assays (Serafini et al. 1996). Evidently, the floor plate provides at least one other chemoattractant in addition to Netrin-1. Surprisingly, this attractant turned out to be Sonic Hedgehog (Shh) (Charron et al. 2003), better known for its function in earlier development as a morphogen that patterns cell fates within the spinal cord (Fig. 2). Shh acts as a chemoattractant through a Src-family-kinasedependent signaling pathway, independent of transcription but dependent on a Patched/ smoothened coreceptor Boc (Okada et al. 2006; Yam et al. 2009).
For the very early phase of their growth in the dorsal spinal cord, commissural axons are also repelled ventrally by signals from the dorsal roof plate. Among these roof-plate repellents are the BMP family proteins BMP7 and growth/ differentiation factor 7 (GDF7) (Augsburger et al. 1999; Butler and Dodd 2003; Yamauchi et al. 2008). Another diffusible protein called Draxin (dorsal repulsive axon guidance protein) also acts as a dorsal midline repellent in vitro, although it may only play a minor role in commissural axon guidance in vivo. In draxin knockout mice, commissural axons still project ventrally from the roof plate, with only mild defasciculation phenotypes (Islam et al. 2009) (Fig. 2).

The role of Netrin family proteins in midline axon guidance has been widely conserved in evolution. In both worms and flies, as in the vertebrate spinal cord, Netrins are expressed at the ventral midline and guide axons along the dorsal-ventral axis. Caenorhabditis elegans has a single Netrin, UNC-6, which is expressed at the ventral midline and required for midline guidance via the DCC family member UNC40 (Hedgecock et al. 1990; Ishii et al. 1992; Chan et al. 1996; Wadsworth et al. 1996). Drosophila has two Netrin genes, both also expressed by midline cells. They have overlapping functions in commissure formation, as only when both genes are deleted is there a significant reduction in the number of axons that cross the midline. (Harris et al. 1996; Mitchell et al. 1996; Brankatschk and Dickson 2006). The same defect is also observed upon loss of fra, which encodes a Netrin receptor of the DCC family (Kolodziej et al. 1996). The fly Dscams also bind Netrins and contribute to commissure formation (Andrews et al. 2008), but the genetic evidence is more consistent with a model in which Dscams act independently of Netrins in commissure formation.

Netrins may also act in a slightly different manner at the fly midline compared to the vertebrate midline. Tethering Drosophila Netrin to the cell membrane does not block its function in commissure formation, suggesting that it does not need to move away from the midline cells that produce it (Brankatschk and Dickson 
2006). Indeed, commissural axons orient normally toward the midline even in embryos lacking both Netrins. These data suggest that the fly Netrins do not act as long-range chemoattractants to draw commissural axons toward the midline, but rather as short-range permissive cues to promote growth across the midline once they get there (Brankatschk and Dickson 2006). Although vertebrate Netrins do diffuse over a hundred microns from the floor plate (Kennedy et al. 2006), they also appear to act as short-range cues in other contexts (Deiner et al. 1997). At the fly midline, there is currently no evidence that Hedgehog, or any other molecule, acts as a long-range attractant for commissural axons, nor have any lateral repellents been identified that might function in a manner similar to the BMPs in vertebrates. The initial medial growth of commissural axons in Drosophila might instead rely on local polarity cues within the nerve cord.

\section{CHOOSING AN IPSILATERAL OR CONTRALATERAL PATHWAY}

Commissural axons, by definition, continue across the midline once they get there. But not all axons that approach the midline eventually cross it. Some remain on their own side to form ipsilateral projections. Genetic studies in Drosophila have helped to explain why some axons cross the midline but others do not. By focusing on identifiable neurons, these studies have examined mutants in which specific axons inappropriately cross or do not cross the midline. A fairly simple logic has emerged, and appears to apply to the midline crossing decision in vertebrates as well. In addition to attractive cues such as the Netrins, the midline also secretes repellent molecules. Axons differ in their sensitivity to these cues. Those that are more sensitive to the attractants cross the midline, whereas those axons that are more sensitive to the repellents remain ipsilateral.

The major midline repellent in Drosophila is Slit, which acts through Robo family receptors (Kidd et al. 1998; Battye et al. 1999; Kidd et al. 1999). Drosophila has one slit gene and three robo genes, called robo1 (or just robo, as it was the founding member of this family), robo2, and robo3 (Rothberg et al. 1988; Kidd et al. 1998; Rajagopalan et al. 2000a; Simpson et al. $2000 \mathrm{~b})$. Robol is the key to the midline crossing decision. It is expressed in most if not all axonal growth cones in the developing central nervous system (Kidd et al. 1998), and in embryos that lack either slit or robol all axons initially grow towards the midline (Seeger et al. 1993; Battye et al. 1999; Kidd et al. 1999). This suggests that repulsion by Slit and Robol serves to keep axons away from the midline, and that commissural axons have some mechanism that allows them to at least transiently overcome this repulsion.

What overrides Slit-Robol repulsion in commissural axons? Genetically, such a mechanism should reveal itself through mutations that result in the opposite phenotype to slit and robol-too few rather than too many axons crossing the midline. The most striking such mutant is commissureless (comm), in which the commissures are almost completely lacking (Seeger et al. 1993; Harris et al. 1996; Kolodziej et al. 1996; Mitchell et al. 1996; Tear et al. 1996). The comm gene is expressed in commissural but not ipsilateral neurons, and is both necessary and sufficient for midline crossing (Bonkowsky et al. 1999; Georgiou and Tear 2002; Keleman et al. 2002; McGovern and Seeger 2003; Keleman et al. 2005; Hiramoto and Hiromi 2006). Double mutants between comm and robol have the robol phenotype, suggesting that comm promotes crossing by antagonizing robo1 (Seeger et al. 1993).

Molecular cloning of the comm gene revealed that it encodes a small transmembrane protein, but did not immediately suggest a molecular mechanism for its role in midline crossing (Tear et al. 1996). Subsequent experiments in heterologous cells in vitro (Keleman et al. 2002; Myat et al. 2002) and in sensory neurons in vivo (Keleman et al. 2005) showed that Comm can act as a sorting receptor to control the intracellular trafficking of Robo1, diverting it from the Golgi to endosomes. This would prevent Robol from reaching the growth cone, and so the transient expression of comm specifically in commissural neurons should render 
B.J. Dickson and Y. Zou

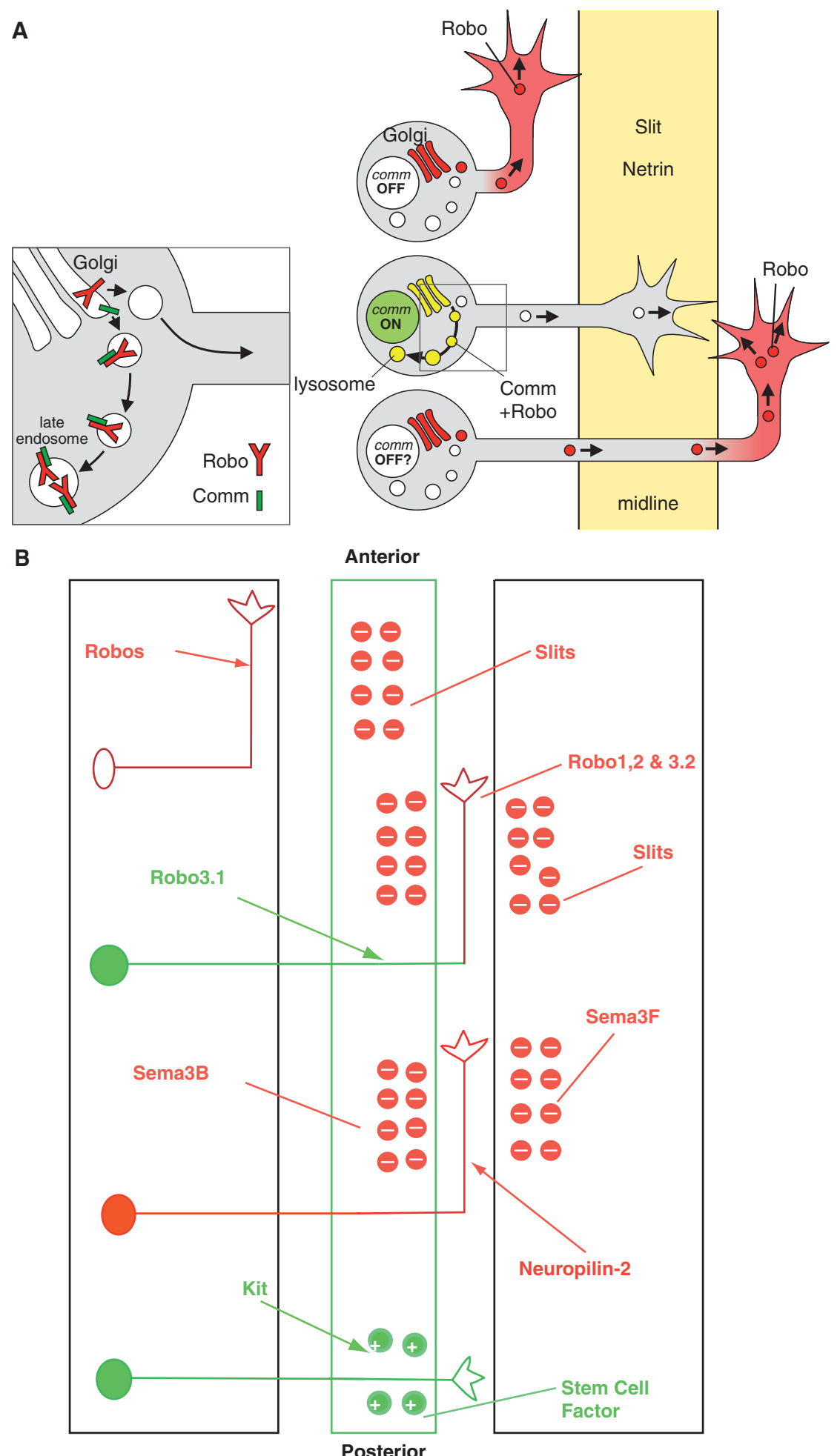

Figure 3. See facing page for legend. 
these axons insensitive to Slit and thereby allow them to cross the midline (Keleman et al. 2002) (Fig. 3A).

This picture is still incomplete. For example, one puzzling finding is that a mutant form of Robo 1 that is largely insensitive to Comm's sorting action in vitro is still functional in vivo (Gilestro 2008). Either a low level of sorting activity suffices in vivo, or Comm can also regulate Robol through some other mechanism. We also do not know what regulates comm expression, turning it on specifically in commissural but not ipsilateral neurons. It is intriguing to note that Frazzled, acting independently of Netrin, contributes to the transcriptional control of comm (Yang et al. 2009), although evidently not to its precise spatial or temporal regulation.

Just as Netrins and their DCC receptors have a conserved role in midline attraction, Slits and their Robo receptors have a conserved role in midline repulsion. The vertebrate floor plate expresses three Slits and commissural axons express Robo receptors. A series of in vivo and in vitro assays using mouse embryos lacking various combinations of these Slit and Robo genes have demonstrated that they have repulsive functions analogous to those in Drosophila (Zou et al. 2000; Long et al. 2004; Chen et al. 2008). Vertebrates do not however have a comm gene. So what antagonizes Slit/Robo signaling in vertebrates to allow commissural axons to cross the floor plate?

The answer came with the surprising finding that knocking out a divergent member of the Robo family, called Rig-1 or Robo3, did not result in the excess crossing phenotype one might expect from loss of a receptor for a midline repellent. Rather, it produced precisely the opposite phenotype of little or no crossing (Sabatier et al. 2004)-reminiscent of the comm mutant phenotype in Drosophila. Exploring this finding further revealed that, just as Comm inhibits Slit/Robol repulsion in Drosophila commissural neurons, a specific splice variant of Robo3, Robo3.1, inhibits Slit/Robo repulsion in vertebrate commissural axons (Chen et al. 2008; Sabatier et al. 2004). The mechanism of this inhibition, however, is clearly different. Robo3.1 does not regulate the intracellular trafficking of Robo receptors, but may instead interfere with Robo's ability to transduce a repulsive signal in the growth cone in response to Slit stimulation (Sabatier et al. 2004) (Fig. 3B).

Intriguingly, one of the fly Robos might have a similar positive function in midline crossing. Mutations in the robo2 gene have, on their own, only a mild effect on midline axon guidance. But in a Netrin or fra mutant background, in which many commissures still form, loss of robo2 function eliminates almost all of these residual commissures (Spitzweck et al. 2010). Thus, at least in the absence of Netrin signaling, Robo2, like vertebrate Rig-1, has a critical positive function in commissure formation. The mechanism underlying this positive function of Robo2 is unclear, but it might involve inhibition of Robo1 signaling in a manner similar to that of

Figure 3. Molecular cues controlling the pathway choices at the Drosophila and vertebrate midline. (A) Comm regulates Robo1 trafficking and midline crossing. Model for the sorting action of Comm on Robo1; inset to the left shows enlarged view of the boxed region. In an ipsilateral neuron (top), Comm is absent and Robol is inserted into the growth cone, ensuring midline repulsion by Slit. In a commissural neuron (middle), Comm is present and diverts Robol to the endosomes, making the growth cone insensitive to Slit. After crossing (bottom), Comm is reduced and Robol begins to accumulate in the contralateral axon segment, potentially preventing the growth cone from crossing again. (Adapted from Dickson and Gilestro 2006.) (B) The Slit/ Robo and Sema3B, 3F/Neuropilin2 systems control midline guidance. Robo3.1 (Rig-1), an inhibitor of Robol and 2, is required for commissural axons to cross the midline. In the absence of Robo3.1, no commissural axons are able to cross the floor plate, because Slits in the midline repel commissural axons. After entering the floor plate, commissural axons acquire a repulsive response to Sema3B (in the floor plate), Sema3F (in the ventral spinal cord gray matter) and Slits (in the floor plate and ventral spinal cord gray matter), which turn their trajectory into a longitudinal one. The stem cell factor in the floor plate promotes the growth of commissural axons via the Kit receptor to allow axons to cross the midline in this highly repulsive environment. 
Rig-1. If this were the case, then in both vertebrates and flies, a divergent Robo would be specialized as an anti-Robo to promote rather than inhibit midline crossing. Whereas in flies any role of an anti-Robo is subordinate to that of Comm, a recent innovation in Drosophila evolution, the anti-Robo in vertebrates takes on the main responsibility for inhibiting Slit repulsion to allow commissure formation.

\section{MOVING ON}

If commissural axons find the midline so attractive, why do they leave it again as soon as they get there? What drives them out on the contralateral side? In principle, commissural axons might either lose attraction or gain repulsion once they reach the midline. For vertebrates, there is direct evidence for both a loss of attraction (Shirasaki et al. 1998) and a gain of repulsion (Zou et al. 2000). For Drosophila, the evidence is less direct, and has been interpreted as a gain of repulsion (Kidd et al. 1998).

In the vertebrate hindbrain, commissural axons originating from the dorsal cerebellar plate no longer respond to Netrin-1 attraction after midline crossing (Shirasaki et al. 1998). Similarly, commissural axons in the spinal cord lose attraction to both Netrin-1 and Shh after crossing (Lyuksyutova et al. 2003). Conversely, factors such as Slits and the secreted semaphorins Sema3B and Sema3F, which have no effect on commissural axons before crossing, become potent repellents after crossing (Zou et al. 2000) (Fig. 3B). In the chick, the attractant Shh may even become a repellent for commissural axons upon midline crossing (Bourikas et al. 2005). And finally, in the mouse spinal cord, commissural axons also become sensitive to Stem Cell Factor (SCF) as they cross the floor plate, which acts as through its receptor, Kit, to stimulate outgrowth and provide an extra boost to further promote midline exit (Gore et al. 2008).

How do commissural axons switch their responses to all these signals so dramatically as they cross the floor plate? Studies using Xenopus spinal neurons in vitro have suggested that the down-regulation of Netrin-1 attraction could be mediated by a hierarchical interaction among guidance signals, in which Slit-1 silences the attractive effect of Netrin-1 (but not its ability to stimulate growth) (Stein and Tessier-Lavigne 2001). Slit-activated Robo binds DCC via the cytoplasmic domain and inhibits DCC signaling, and adding Slit completely blocks the turning of isolated spinal axons toward a Netrin-1 source (Stein and Tessier-Lavigne 2001). Because Slits are highly enriched in the floor plate, they are well placed to silence Netrin-1 responsiveness in commissural axons in vivo, though this remains to be fully tested.

The repulsive response to semaphorins after crossing may be switched on through an interaction with Shh (Parra and Zou 2010). Shh can induce repulsive response of precrossing commissural axons, which have not contacted the midline, to Sema3B and Sema3F, in an explant assay. Blocking Shh function in commissural neurons in a spinal cord culture either with a Shh antibody, a constitutive inhibitory (with Shh binding domain deleted) form of Patched-1, or a Smoothened (Smo) shRNA construct resulted in midline stalling, floor plate recrossing, and overshooting after crossing. These phenotypes are all reminiscent of those phenotypes found in mutant mouse embryos lacking Neuropilin-2, a receptor for both Sema3B and Sema3F, and are thus consistent with a role for Shh in conferring sensitivity to semaphorins (Zou et al. 2000). This Shh-mediated switch mechanism is at least in part mediated by the ability of Shh to reduce cAMP levels in commissural axons. Enhancing cAMP levels in precrossing axons diminished Shh-induced Semaphorin repulsion and caused midline stalling and overshooting/wandering of postcrossing axons (Parra and Zou 2010).

Gaining sensitivity to both Sema3B and Sema3F is critical for the guidance of postcrossing commissural axons. Sema3B is expressed in the floor plate, and upon crossing commissural axons also become sensitive to this midline repellent in addition to Slit. These two repellents may act on different subsets of commissural axons, or contribute in different ways to the distinct guidance decisions of midline entry and midline exit. Interestingly, Sema3F is not 
expressed at the midline, but rather in the gray matter. Thus, although Sema3B likely drives commissural axons out of the midline, Sema3F could constrain their trajectories to a permissive corridor along the opposite side (Zou et al. 2000; Long et al. 2004; Chen et al. 2008) (Fig. 3). Indeed, another common defect in Neuropilin-2 mutant embryos and in cultured rat spinal cords where Shh signaling is inhibited is overshooting of commissural axons into the contralateral ventral spinal cord (Zou et al. 2000; Long et al. 2004; Chen et al. 2008; Parra and Zou 2010). It will be interesting to further test Shh effects on semaphorin signaling in various genetic mouse models.

Although there is no direct evidence that Drosophila commissural axons change their responsiveness to midline guidance cues upon crossing in the same way that vertebrate commissural axons do (Fig. 2A), comm mRNA goes down as commissural axons cross (Keleman et al. 2002) and Robol protein, previously absent, becomes enriched on the axon segment that extends beyond the midline (Kidd et al. 1998). Thus, by down-regulating Comm and thereby up-regulating Robo1, commissural axons could potentially switch their response to the midline from attraction to repulsion. It has been suggested that might explain why these axons leave the midline and never cross again (Kidd et al. 1998).

This model might seem to follow logically from the roles of Comm and Robol in the initial crossing decision. But the mechanisms that ensure the temporally distinct responses of post- and precrossing commissural axons are not necessarily the same as those that ensure the spatially distinct responses of commissural and ipsilateral axons. Indeed, some observations are difficult to reconcile with the notion that down-regulation of Comm and up-regulation of Robol are critical to keep commissural axons moving on once they reach the midline. For example, forcing persistent comm expression in specific ipsilateral neurons diverts them into a commissural pathway, but it does not cause them to stall at the midline or to cross repeatedly (Bonkowsky et al. 1999; Keleman et al. 2005). Assuming that persistent Comm keeps Robol levels low in these axons, this result also suggests that Robol is not required in single postcrossing axons to prevent them from recrossing. Also, like Robo1, Robo2 and Robo3 proteins, as well as the Comm-insensitive variant of Robo1, are all selectively enriched on the postcrossing axon segments-but for these Robo proteins at least, this distribution does not depend on Comm at all, let alone its precise temporal regulation (Rajagopalan et al. 2000a,b; Gilestro 2008).

If Comm down-regulation is not the key to understanding how Robol becomes enriched on post-crossing axons, and what keeps them from crossing again, then what is? One alternative idea posits that, instead of intrinsic regulation by Comm, it could be extrinsic cues at the midline that lead to a down-regulation of Robol. This would create a positive feedback loop owing to mutual inhibition of high Robol levels and midline crossing (Dickson and Gilestro 2006). Such mutual inhibition creates a bistable switch, characteristic of all-or-none decisions in development. The actual outcome of such a system can then be determined by any number of inputs external to the feedback loop itself. For precrossing commissural axons, the initial bias that favors growth toward the midline might be set by Comm, acting to reduce Robol levels. For post-crossing axons, the switch might be reset to favor growth away from the midline (and thus high Robol) simply by the natural tendency of axons to grow straight ahead in a permissive environment, and perhaps also through fasciculation with the contralateral homolog (Myers and Bastiani 1993).

\section{MAKING ANTERIOR-POSTERIOR GUIDANCE DECISIONS}

Forming an orthogonal array of axon pathways at the midline not only requires directing axon traffic along the dorsal-ventral or mediallateral axis, but also along the anterior-posterior axis. For many vertebrate and Drosophila commissural axons, this involves making the correct anterior or posterior turn after crossing. For Drosophila commissural axons, it also involves the choice of crossing the midline in 


\section{B.J. Dickson and Y. Zou}

either of the two commissures in each segment-the anterior commissure (AC) or posterior commissure (PC).

For Drosophila commissural axons, the choice between the AC and PC is probably determined in part by the position of the cell body, with many axons simply crossing in the closest commissure. In fact, the two commissures only become fully resolved after the first axons have crossed, when some of the midline glia migrate to nestle in between the $\mathrm{AC}$ and the PC. But for some commissural axons, choosing the correct commissure also relies on specific molecular signals that discriminate the two pathways. One such signal is the secreted protein Wnt protein Wnt5 (Yoshikawa et al. 2003). Wnt5 is predominantly expressed by cells near the PC and appears to act as a repellent signal that keeps $\mathrm{AC}$ axons out of the $\mathrm{PC}$ (Yoshikawa et al. 2003) (Fig. 4A). If Wnt5 is missing, some AC axons switch over to the PC. Conversely, if Wnt5 is ectopically expressed along the entire midline, the AC completely fails to form. In both cases, the PC is normal. The
A

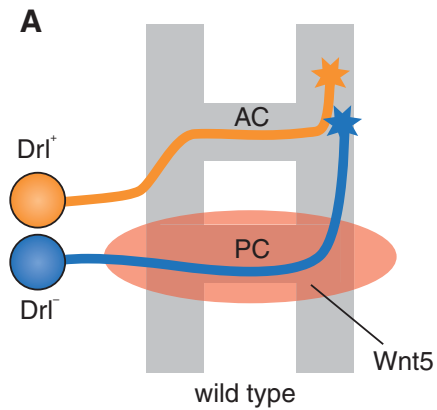

D

Spinal cord

commissural neurons

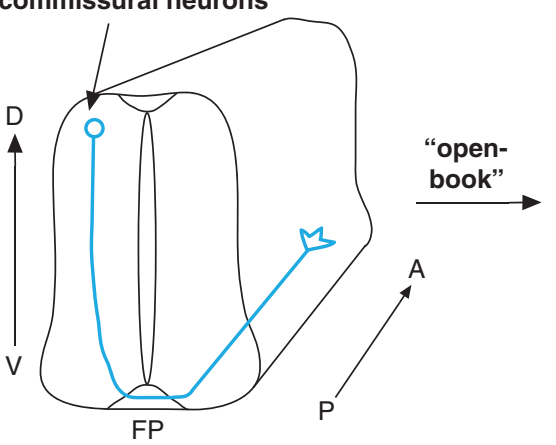

B

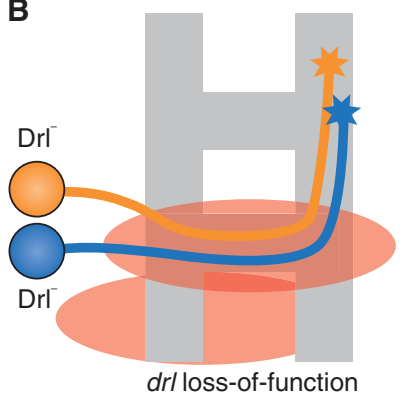

E
C

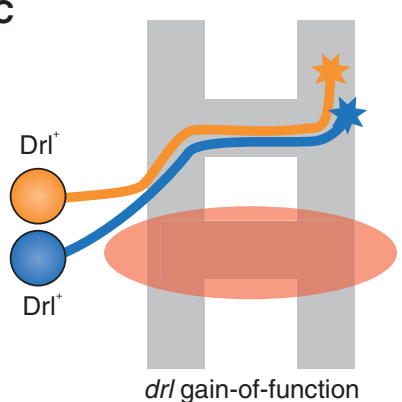

$\mathbf{F}$
Frizzled3-/- or Wnt gradient disrupted

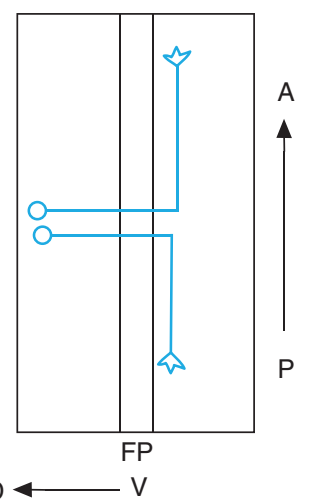

Figure 4. Wnts organize axon connections along the A-P axis at both the Drosophila and vertebrate midline. (A$C$ ) Wnt5 and Drl regulate the choice of anterior or posterior commissure. AC axons (orange) express Drl, PC axons (blue) do not. Wnt5 accumulates on the PC and repels axons expressing Drl. These phenotypes are only partially penetrant. $(D)$ Trajectory of the dorsal spinal cord commissural axons along the dorsal-ventral (D-V) and anterior-posterior (A-P) axes in a transverse view; (E) Spinal cord is splayed open along the dorsal midline, showing the $90^{\circ}$ anterior turning right after midline (floor plate) crossing. Several Wnts are expressed at differential levels along the A-P axis in the floor plate (FP) and broadly in the spinal cord with an anterior-high posterior-low gradient. Frizzled3 is expressed in commissural neurons; $(F)$ Randomized turning along the A-P axis when Wnt gradient is disrupted in the open-book explants by Wnt inhibitors (sFRPs), Wnt4-secreting cell clumps positioned caudal to the explants or in Frizzled 3 knockout mice (Lyuksyutova et al. 2003). 
Wnt5 receptor on AC growth cones is Derailed (Drl), a member of the Ryk family of atypical receptor tyrosine kinases (Bonkowsky et al. 1999; Yoshikawa et al. 2003). Drl is exclusively expressed on AC axons, and if it is lacking some of these axons cross in the PC (Fig. 4B). $\mathrm{Drl}$ is not expressed on PC axons, explaining why they do not seem to care about the presence or absence of Wnt5. If, however, PC axons are forced to express Drl, some of them now cross instead in the AC (Fig. 4C).

The anterior or posterior turning of commissural axons after crossing has been less well studied in Drosophila, but has been intensively investigated in the vertebrate system. Here too, Wnt proteins play a critical role, in this case acting as attractants rather than repellents. A population of commissural axons originating from the dorsal spinal cord turns anteriorly after midline crossing (Fig. 4D). These commissural axons are not sensitive to Wnts before crossing, but respond to them once they arrive at the contralateral border of the floor plate (Lyuksyutova et al. 2003). Several Wnts, including Wnt4, Wnt7b, Wnt7a, and Wnt5a, are expressed in an anterior-posterior decreasing gradient along the spinal cord at the ventral midline and attract postcrossing commissural axons to turn anteriorly. If the Wnt gradient is disrupted by adding Wnt inhibitors, secreted Frizzled-related proteins (sFRPs), or by positioning Wnt4-secreting cell aggregates in open-book explant culture, commissural axons show specific defects in A$\mathrm{P}$ growth after midline crossing (Fig. 4E,F). Moreover, in Frizzled3 mutant embryos, spinal cord commissural axons lose A-P directionality in vivo (Fig. 4F) (Lyuksyutova et al. 2003). aPKC, a kinase, central to cell polarity signaling, is required cell autonomously for postcrossing commissural axons to be attracted to Wnt4 and to turn anteriorly in open-book explant assays (Wolf et al. 2008). Moreover, Wnts are highly conserved A-P guidance cues, because in C. elegans, Wnt signaling controls the direction of axonal turning and cell polarization along the A-P axis (Pan et al. 2006; Hilliard and Bargmann 2006; Prasad and Clark 2006).

Another factor that may contribute to anterior-posterior guidance decisions in vertebrates is, once again, Shh. In the chick, Shh is expressed in an anterior-low to posterior-high gradient in the floor plate and repels commissural axons after midline crossing. RNAi knockdown of Shh caused A-P guidance defects in chick commissural axons (Bourikas et al. 2005). It remains to be tested whether Shh is expressed in the similar A-P gradient in the rodent spinal cord and whether it also controls A-P guidance of commissural axons in mice and rats. If so, it would also be important to know whether the Shh pathway collaborates with the Wnt signaling pathway, or whether Wnts and Shh regulate the $\mathrm{A}-\mathrm{P}$ guidance of different subpopulations of commissural axons.

\section{FORMING LONGITUDINAL PATHWAYS}

There is currently no evidence that longitudinal axons in Drosophila rely on long-range gradients of Wnts or any other factors for their anterior or posterior guidance. Most likely, these decisions in Drosophila, like the choice of anterior or posterior commissure, rely on local cues in each segment. One consequence of having segmentally repeated guidance cues is that longitudinal axons will repeatedly encounter the same cues as they extend from segment to segment along the nerve cord. Just as commissural axons must respond differently to midline cues before and after crossing, longitudinal axons might then need to respond to segmental cues differently in their own segment than in all others. A possible instance of this phenomenon has been documented for the ipsilateral dMP2 axon, which extends posteriorly to pioneer a longitudinal pathway close to the midline (Fig. 1D).

As an ipsilateral neuron, dMP2 expresses Robol but not Comm. Robol is needed to prevent dMP2 from crossing, but may also have a second role in helping it extend longitudinally into the next segment. As it reaches the next segment, the dMP2 growth cone encounters a lateral patch of Netrin. This Netrin is evidently derived from midline cells, but may have been transported laterally by the movement of commissural axons (Hiramoto et al. 2000). dMP2 normally ignores this Netrin patch, extending 
across it and into the next segment. However, in the absence of Robo1, dMP2 instead responds ectopically to Netrin and is diverted medially from its longitudinal trajectory (Hiramoto and Hiromi 2006). One potential explanation for this behavior is that the same Slit-Robo1 signal that initially prevents midline crossing also normally renders dMP2 insensitive to Netrin (Hiramoto and Hiromi 2006) - a model that draws heavily on the proposed hierarchical interaction between Slit-Robo and Netrin-DCC signaling in vertebrate commissural axons (Stein and Tessier-Lavigne 2001).

As they project along these longitudinal pathways, axons select between one of several discrete axon fascicles, each spaced a characteristic distance from the midline (Raper et al. 1983a; Raper et al. 1983b) (Fig. 5). Cell ablation experiments initially demonstrated that axonaxon interactions are critical for pathway selection, formulated as the labeled pathways hypothesis (Bastiani et al. 1984; Raper et al. 1984). This hypothesis posits that each fascicle is labeled by a unique combination of cell surface markers, and that individual axons choose a fascicle by reading these markers. Molecular approaches have provided candidate pathway labels, including the immunoglobulin family member Fasciclin II (Fas II) and semaphorin Semala (Fas IV), for which subsequent genetic studies defined their roles as attractive and repulsive guidance cues, respectively (Bastiani et al. 1984; Bastiani et al. 1987; Patel et al. 1987; Kolodkin et al. 1992; Lin et al. 1994; Yu et al. 1998).

At a coarser level, lateral pathway selection is also controlled by the Robo proteins (Rajagopalan et al. 2000b; Simpson et al. 2000a). The three Robos, Robo1, Robo2, and Robo3, define three broad zones within the longitudinal connectives: a medial zone occupied by axons that express Robol alone, an intermediate zone of axons that express both Robo1 and Robo3, and a lateral zone of axons that express all three Robo proteins. This so-called Robo code is instructive for lateral pathway selection: if Robo2 or Robo3 is absent, lateral axons are shifted closer to the midline (Fig. 5A,B). Conversely, if either Robo2 or Robo3 is misexpressed in medial axons, then these axons are displaced laterally.

Recent experiments have examined more subtle manipulations of the Robo code, with somewhat different results (Spitzweck et al. 2010). Rather than eliminating a Robo protein completely, or overexpressing it at high levels, these experiments selectively replaced one Robo protein by another, but still expressing it in the same spatial and temporal pattern as the Robo it replaced. Surprisingly, under these circumstances lateral pathway selection appears remarkably normal. Thus, it is not the specific combination of Robo receptors that matters, but where and when they are expressed-the distinct lateral zones arise through differences in the timing, location, and levels of gene expression, as encoded in the cis-regulatory elements of the three robo genes.

There is some evidence, albeit still rather limited, that Robo receptors also contribute to the formation of discrete zones within the lateral funiculus of the mouse spinal cord (Long et al. 2004). These longitudinal axons are segregated into a Robol-positive medial zone, a Robo2-positive lateral zone, and a mixed intermediate zone (Fig. 5C,D). In Robo1-/- embryos, the medial region is reduced and the lateral region thicker, suggesting a lateral shift of the Robo2-positive axons. Conversely, the medial region is thicker in Robo2-/- embryos and the lateral region thinner, due in part to a medial shift of Robo1-positive axons.

\section{CONCLUSIONS AND PERSPECTIVES}

Tremendous progress has been made over the past couple of decades in understanding both the logic and mechanisms of axon pathfinding at the CNS midline. This work, primarily using Drosophila and mice as models, has yielded both rich details and broader general principles. But it has also left many open questions. Here, we have only been able to touch on some of the key experimental findings. Now, in closing, we turn to consider some of the general principles that have emerged, and the critical questions that remain. 
Navigating Intermediate Targets: The Nervous System Midline

A

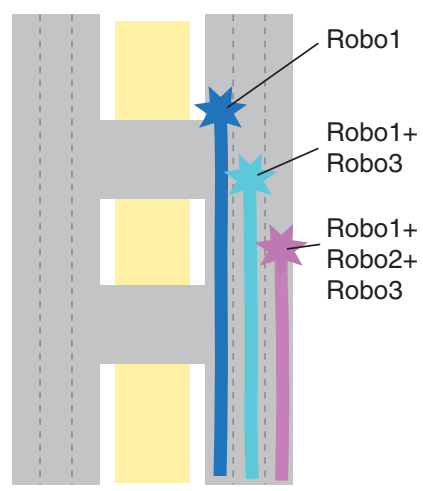

C

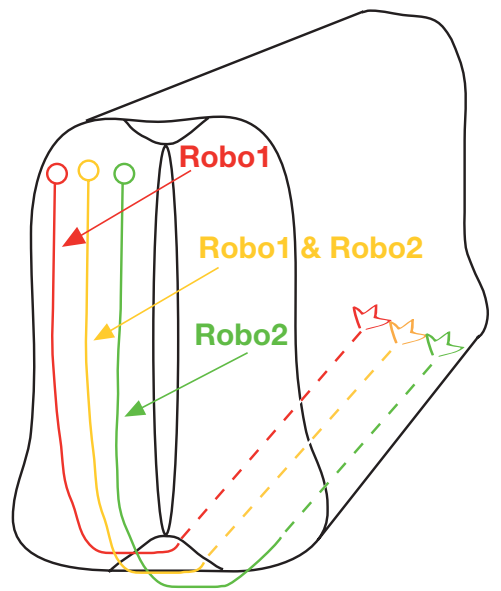

B

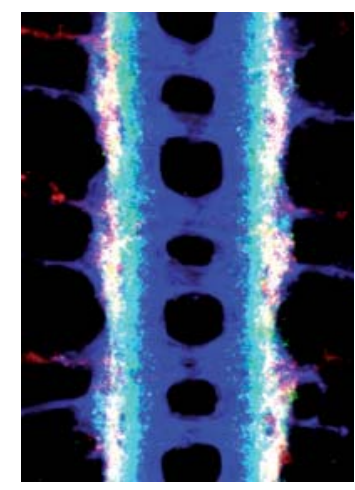

D

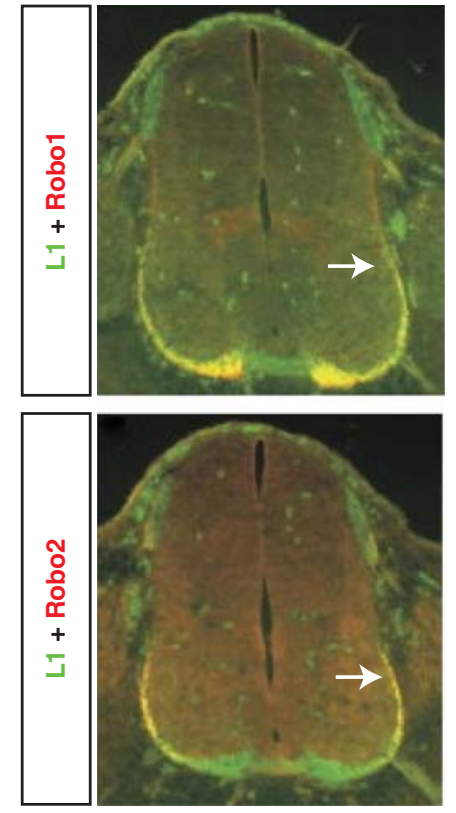

Figure 5. A Robo code for lateral positioning. (A) Longitudinal tracts are divided into three zones, with axons expressing Robo1 in the medial zone, axons expressing Robol and Robo3 in the intermediate zone, and axons expressing all three Robos in the lateral zone. (B) Confocal image of a nerve cord, stained for all axons (blue), Robo3 (green), and Robo2 (red). Image in $B$ is from Rajagopalan et al. 2000b. $(C)$ In rodent spinal cord, commissural axons expressing Robol project in the ventral funiculus after midline crossing, those expressing Robo2 take a more lateral position in the lateral funiculus and those expressing both occupy the longitudinal tracts in between. $(D)$ Fluorescence images of Robo1 and Robo2 staining in the ventral and lateral funiculus. Image in $D$ is from Long et al. 2004.

One key feature of the midline-common to both vertebrates and invertebrates, and likely to apply to other intermediate targets as well-is that it provides both attractive and repulsive cues for growing axons. These guidance cues organize axonal trajectories along both the perpendicular and parallel axes. Distinct axons follow distinct paths because they express distinct combinations of receptors for these guidance cues, and because they change their sensitivity to these cues over time. Changing sensitivity to guidance cues is particularly 
critical for axons that grow directly toward the intermediate target, as it allows them to move on once they reach it. Precise temporal regulation of guidance responses may also be important for axons that grow parallel to the intermediate target, ensuring that any turns are made at the right point. As a general rule, these dynamic growth cone responses rely on posttranscriptional mechanisms, including hierarchical interactions between the guidance molecules themselves.

The molecular tool-kit for axon pathfinding at the CNS midline is both evolutionarily ancient and highly versatile. The Netrin/DCC and Slit/Robo systems were evidently laid down as midline attractants and repellents in the earliest bilaterally symmetric nervous systems. Similarly, Wnt proteins may have an ancient and conserved role in anterior-posterior guidance. Over the course of evolution, interesting variations have arisen on these themes. This includes for example subtle differences in the way Netrins and Wnts operate in insects and vertebrates, as well as the evolution of distinct negative regulators of Slit/Robo repulsion. Constructing the more complex vertebrate nervous systems also involves additional midline attractants and repellents, such as Shh and the semaphorins, which have either been gained during vertebrate evolution or lost during insect evolution.

What are the key open questions? One is the role of long-range gradients in axon pathfinding. Such gradients have been particularly popular in theoretical models of axon guidance, and are frequently invoked to explain the action of midline guidance molecules. Indeed, for many of these molecules, their mutant phenotypes and in vitro properties are consistent with this view. Yet direct evidence for molecular gradients in vivo has generally been hard to come by. Understanding how gradients of guidance molecules form in vivo, and how growth cones detect and respond to these gradients, is an important challenge for the future.

There is also still much to be done to understand the dynamic events within the growth cone. How do growth cones respond, from moment to moment, to minor fluctuations in the levels and combinations of guidance cues that they encounter along their journey? And how do they change these responses over time, so that they make the right turn at the right time and place? Too much of our current understanding of growth cone behavior is derived from the analysis of fixed embryos, or from watching live axons grow in artificial in vitro environments. Neither captures the dynamics of navigation in a complex multicellular environment. Moreover, too much of our current understanding is based on relatively classic genetic and biochemical studies, neither of which provides the spatial or temporal resolution needed to resolve these dynamic events. Imaging the cellular and molecular processes at work in navigating growth cones in vivo will be challenging. But advances in molecular imaging are bringing this possibility within reach. Applying these methodologies to midline axon guidance will almost certainly overturn some of the models presented here, and lead us to many new surprises and deeper insights. The midline still has many secrets to reveal. It promises to remain an attractive and insightful model for investigating the logic and mechanisms of axon pathfinding for some time to come.

\section{REFERENCES}

Andrews GL, Tanglao S, et al. 2008. Dscam guides embryonic axons by Netrin-dependent and -independent functions. Development 135: 3839-3848.

Augsburger A, Schuchardt A, et al. 1999. BMPs as mediators of roof plate repulsion of commissural neurons. Neuron 24: $127-141$.

Bastiani MJ, Harrelson AL, et al. 1987. Expression of fasciclin I and II glycoproteins on subsets of axon pathways during neuronal development in the grasshopper. Cell 48: 745-755.

Bastiani MJ, Raper JA, et al. 1984. Pathfinding by neuronal growth cones in grasshopper embryos. III. Selective affinity of the G growth cone for the P cells within the A/P fascicle. J Neurosci 4: 2311-2328.

Battye R, Stevens A, et al. 1999. Axon repulsion from the midline of the Drosophila CNS requires slit function. Development 126: 2475-2481.

Bonkowsky JL, Yoshikawa S, et al. 1999. Axon routing across the midline controlled by the Drosophila Derailed receptor. Nature 402: 540-544.

Bourikas D, Pekarik V, et al. 2005. Sonic hedgehog guides commissural axons along the longitudinal axis of the spinal cord. Nat Neurosci 8: 297-304. 
Brankatschk M, Dickson BJ. 2006. Netrins guide Drosophila commissural axons at short range. Nat Neurosci 9: 188-194.

Butler SJ, Dodd J. 2003. A role for BMP heterodimers in roof plate-mediated repulsion of commissural axons. Neuron 38: $389-401$.

Chan SS, Zheng H, et al. 1996. UNC-40, a C. elegans homo$\log$ of DCC (Deleted in Colorectal Cancer), is required in motile cells responding to UNC-6 netrin cues. Cell 87: 187-195.

Charron F, Stein E, et al. 2003. The morphogen sonic hedgehog is an axonal chemoattractant that collaborates with netrin-1 in midline axon guidance. Cell 113: 11-23.

Chen Z, Gore BB, et al. 2008. Alternative splicing of the Robo3 axon guidance receptor governs the midline switch from attraction to repulsion. Neuron 58: 325-332.

Deiner MS, Kennedy TE, et al. 1997. Netrin-1 and DCC mediate axon guidance locally at the optic disc: loss of function leads to optic nerve hypoplasia. Neuron 19: 575-589.

Dickson BJ, Gilestro GF. 2006. Regulation of commissural axon pathfinding by slit and its Robo receptors. Annu Rev Cell Dev Biol 22: 651-675.

Georgiou M, Tear G. 2002. Commissureless is required both in commissural neurones and midline cells for axon guidance across the midline. Development 129: 2947-2956.

Gilestro GF. 2008. Redundant mechanisms for regulation of midline crossing in Drosophila. PLoS ONE 3: e3798.

Gore BB, Wong KG, et al. 2008. Stem cell factor functions as an outgrowth-promoting factor to enable axon exit from the midline intermediate target. Neuron 57: 501-510.

Harris R, Sabatelli LM, et al. 1996. Guidance cues at the Drosophila CNS midline: identification and characterization of two Drosophila Netrin/UNC-6 homologs. Neuron 17: 217-228.

Hedgecock EM, Culotti JG, et al. 1990. The unc-5, unc-6, and $u n c-40$ genes guide circumferential migrations of pioneer axons and mesodermal cells on the epidermis in C. elegans. Neuron 4: 61-85.

Hilliard MA, Bargmann CI. 2006. Wnt Signals and Frizzled activity orient anterior-posterior axon outgrowth in C. elegans. Dev Cell 10: 379-90.

Hiramoto M, Hiromi Y. 2006. ROBO directs axon crossing of segmental boundaries by suppressing responsiveness to relocalized Netrin. Nat Neurosci 9: 58-66.

Hiramoto M, Hiromi Y, et al. 2000. The Drosophila Netrin receptor Frazzled guides axons by controlling Netrin distribution. Nature 406: 886-889.

Ishii N, Wadsworth WG, et al. 1992. UNC-6, a lamininrelated protein, guides cell and pioneer axon migrations in C. elegans. Neuron 9: 873-881.

Islam SM, Shinmyo Y, et al. 2009. Draxin, a repulsive guidance protein for spinal cord and forebrain commissures. Science 323: 388-393.

Keino-Masu K, Masu M, et al. 1996. Deleted in Colorectal Cancer (DCC) encodes a netrin receptor. Cell 87: 175-185.

Keleman K, Rajagopalan S, et al. 2002. Comm sorts Robo to control axon guidance at the Drosophila midline. Cell 110: $415-427$.
Keleman K, Ribeiro C, et al. 2005. Comm function in commissural axon guidance: Cell-autonomous sorting of Robo in vivo. Nat Neurosci 8: 156-163.

Kennedy TE, Serafini T, et al. 1994. Netrins are diffusible chemotropic factors for commissural axons in the embryonic spinal cord. Cell 78: 425-35.

Kennedy TE, Wang H, et al. 2006. Axon guidance by diffusible chemoattractants: a gradient of netrin protein in the developing spinal cord. J Neurosci 26: 8866-8874.

Kidd T, Bland KS, et al. 1999. Slit is the midline repellent for the Robo receptor in Drosophila. Cell 96: 785-794.

Kidd T, Brose K, et al. 1998. Roundabout controls axon crossing of the CNS midline and defines a novel subfamily of evolutionarily conserved guidance receptors. Cell 92: 205-215.

Kolodkin AL, Matthes DJ, et al. 1992. Fasciclin IV: sequence, expression, and function during growth cone guidance in the grasshopper embryo. Neuron 9: 831-845.

Kolodziej PA, Timpe LC, et al. 1996. Frazzled encodes a Drosophila member of the DCC immunoglobulin subfamily and is required for CNS and motor axon guidance. Cell 87: 197-204.

Lin DM, Fetter RD, et al. 1994. Genetic analysis of Fasciclin II in Drosophila: defasciculation, refasciculation, and altered fasciculation. Neuron 13: 1055-1069.

Liu G, Li W, et al. 2009. DSCAM functions as a netrin receptor in commissural axon pathfinding. Proc Natl Acad Sci 106: $2951-2956$

Long H, Sabatier C, et al. 2004. Conserved roles for Slit and Robo proteins in midline commissural axon guidance. Neuron 42: 213-223.

Ly A, Nikolaev A, et al. 2008. DSCAM is a netrin receptor that collaborates with DCC in mediating turning responses to netrin-1. Cell 133: 1241-1254.

Lyuksyutova AI, Lu CC, et al. 2003. Anterior-posterior guidance of commissural axons by Wnt-frizzled signaling. Science 302: 1984-1988.

McGovern VL, Seeger MA. 2003. Mosaic analysis reveals a cell-autonomous, neuronal requirement for Commissureless in the Drosophila CNS. Dev Genes Evol 213: 500-504.

Mitchell KJ, Doyle JL, et al. 1996. Genetic analysis of Netrin genes in Drosophila: Netrins guide CNS commissural axons and peripheral motor axons. Neuron 17: 203-215.

Myat A, Henry P, et al. 2002. Drosophila Nedd4, a ubiquitin ligase, is recruited by Commissureless to control cell surface levels of the roundabout receptor. Neuron 35: 447-459.

Myers PZ, Bastiani MJ. 1993. Growth cone dynamics during the migration of an identified commissural growth cone. J Neurosci 13: 127-143.

Okada A, Charron F, et al. 2006. Boc is a receptor for sonic hedgehog in the guidance of commissural axons. Nature 444: 369-373.

Pan CL, Howell JE, et al. 2006. Multiple Wnts and Frizzled receptors regulate anteriorly directed cell and growth cone migrations in Caenorhabditis elegans. Dev Cell 10: 367-377.

Parra LM, Zou Y. 2010. Sonic hedgehog induces response of commissural axons to Semaphorin repulsion during midline crossing. Nat Neurosci 13: 29-35. 


\section{B.J. Dickson and Y. Zou}

Patel NH, Snow PM, et al. 1987. Characterization and cloning of fasciclin III: A glycoprotein expressed on a subset of neurons and axon pathways in Drosophila. Cell 48: 975-988.

Prasad BC, Clark SG. 2006. Wnt signaling establishes anteroposterior neuronal polarity and requires retromer in C. elegans. Development 133: 1757-1766.

Rajagopalan S, Nicolas E, et al. 2000a. Crossing the midline: Roles and regulation of Robo receptors. Neuron 28: 767-777.

Rajagopalan S, Vivancos V, et al. 2000b. Selecting a longitudinal pathway: Robo receptors specify the lateral position of axons in the Drosophila CNS. Cell 103: 1033-1045.

Ramón y Cajal S. 1995. Histology of the nervous system of man and vertebrates. New York, Oxford University Press.

Raper JA, Bastiani M, et al. 1983. Pathfinding by neuronal growth cones in grasshopper embryos. I. Divergent choices made by the growth cones of sibling neurons. J Neurosci 3: 20-30.

Raper JA, Bastiani M, et al. 1983. Pathfinding by neuronal growth cones in grasshopper embryos. II. Selective fasciculation onto specific axonal pathways. J Neurosci 3: $31-41$.

Raper JA, Bastiani MJ, et al. 1984. Pathfinding by neuronal growth cones in grasshopper embryos. IV. The effects of ablating the $\mathrm{A}$ and $\mathrm{P}$ axons upon the behavior of the $\mathrm{G}$ growth cone. J Neurosci 4: 2329-2345.

Rothberg JM, Hartley DA, et al. 1988. slit: an EGFhomologous locus of D. melanogaster involved in the development of the embryonic central nervous system. Cell 55: 1047-1059.

Sabatier C, Plump AS, et al. 2004. The divergent Robo family protein Rig-1/Robo3 is a negative regulator of slit responsiveness required for midline crossing by commissural axons. Cell 117: 157-69.

Seeger M, Tear G, et al. 1993. Mutations affecting growth cone guidance in Drosophila: genes necessary for guidance toward or away from the midline. Neuron 10: 409-426.

Serafini T, Colamarino SA, et al. 1996. Netrin-1 is required for commissural axon guidance in the developing vertebrate nervous system. Cell 87: 1001-1014.

Serafini T, Kennedy TE, et al. 1994. The netrins define a family of axon outgrowth-promoting proteins homologous to C. elegans UNC-6. Cell 78: 409-424.

Shirasaki R, Katsumata R, et al. 1998. Change in chemoattractant responsiveness of developing axons at an intermediate target. Science 279: 105-107.
Simpson JH, Bland KS, et al. 2000a. Short-range and longrange guidance by Slit and its Robo receptors: a combinatorial code of Robo receptors controls lateral position. Cell 103: 1019-1032.

Simpson JH, Kidd T, et al. 2000b. Short-range and longrange guidance by Slit and its Robo receptors. Robo and Robo2 play distinct roles in midline guidance. Neuron 28: 753-766.

Spitzweck B, Brankatschk B, Dickson BJ. 2010. Distinct protein domains and expression patterns confer divergent axon guidance functions for Drosophila Robo receptors. Cell 140: 409-420.

Stein E, Tessier-Lavigne M. 2001. Hierarchical organization of guidance receptors: silencing of netrin attraction by slit through a Robo/DCC receptor complex. Science 291: 1928-1938.

Tear G, Harris R, et al. 1996. commissureless controls growth cone guidance across the CNS midline in Drosophila and encodes a novel membrane protein. Neuron 16: 501-514.

Tessier-Lavigne M, Placzek M, et al. 1988. Chemotropic guidance of developing axons in the mammalian central nervous system. Nature 336: 775-778.

Wadsworth WG, Bhatt H, et al. 1996. Neuroglia and pioneer neurons express UNC-6 to provide global and local netrin cues for guiding migrations in C. elegans. Neuron 16: $35-46$

Wolf AM, Lyuksyutova AI, et al. 2008. Phosphatidylinositol3-kinase-atypical protein kinase $\mathrm{C}$ signaling is required for Wnt attraction and anterior-posterior axon guidance. J Neurosci 28: 3456-3467.

Yam PT, Langlois SD, et al. 2009. Sonic hedgehog guides axons through a noncanonical, Src-family-kinasedependent signaling pathway. Neuron 62: 349-362.

Yamauchi K, Phan KD, et al. 2008. BMP type I receptor complexes have distinct activities mediating cell fate and axon guidance decisions. Development 135: 1119-1128.

Yang L, Garbe DS, et al. 2009. A frazzled/DCC-dependent transcriptional switch regulates midline axon guidance. Science 324: 944-947.

Yoshikawa S, McKinnon RD, et al. 2003. Wnt-mediated axon guidance via the Drosophila Derailed receptor. Nature 422: 583-588.

Yu HH, Araj HH, et al. 1998. The transmembrane Semaphorin Sema I is required in Drosophila for embryonic motor and CNS axon guidance. Neuron 20: 207-220.

Zou Y, Stoeckli E, et al. 2000. Squeezing axons out of the gray matter: a role for slit and semaphorin proteins from midline and ventral spinal cord. Cell 102: 363-375. 


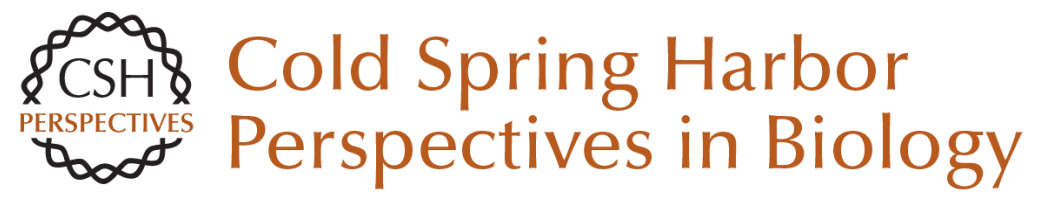

\section{Navigating Intermediate Targets: The Nervous System Midline}

Barry J. Dickson and Yimin Zou

Cold Spring Harb Perspect Biol 2010; doi: 10.1101/cshperspect.a002055 originally published online June 9, 2010

\section{Subject Collection Neuronal Guidance}

Mechanisms and Molecules of Neuronal Wiring: A Primer

Alex L. Kolodkin and Marc Tessier-Lavigne

Guidance Molecules in Axon Pruning and Cell

Death

Pierre Vanderhaeghen and Hwai-Jong Cheng

Initiating and Growing an Axon

F. Polleux and William Snider

Navigating Intermediate Targets: The Nervous System Midline

Barry J. Dickson and Yimin Zou

Cellular Strategies of Axonal Pathfinding

Jonathan Raper and Carol Mason

Guidance Molecules in Axon Regeneration

Roman J. Giger, Edmund R. Hollis II and Mark H. Tuszynski

Signaling from Axon Guidance Receptors Greg J. Bashaw and Rüdiger Klein

Visual Map Development: Bidirectional Signaling, Bifunctional Guidance Molecules, and Competition

David A. Feldheim and Dennis D. M. O'Leary
Wiring the Brain: The Biology of Neuronal

Guidance

Alain Chédotal and Linda J. Richards

Guidance Molecules in Synapse Formation and

Plasticity Kang Shen and Christopher W. Cowan

The Growth Cone Cytoskeleton in Axon

Outgrowth and Guidance

Erik W. Dent, Stephanie L. Gupton and Frank B. Gertler

Topographic Mapping--The Olfactory System Takeshi Imai, Hitoshi Sakano and Leslie B. Vosshall

Self-avoidance and Tiling: Mechanisms of

Dendrite and Axon Spacing Wesley B. Grueber and Alvaro Sagasti

Trafficking Guidance Receptors Bettina Winckler and Ira Mellman

Axon Guidance Molecules in Vascular Patterning Ralf H. Adams and Anne Eichmann

Human Genetic Disorders of Axon Guidance Elizabeth C. Engle

For additional articles in this collection, see http://cshperspectives.cshlp.org/cgi/collection/

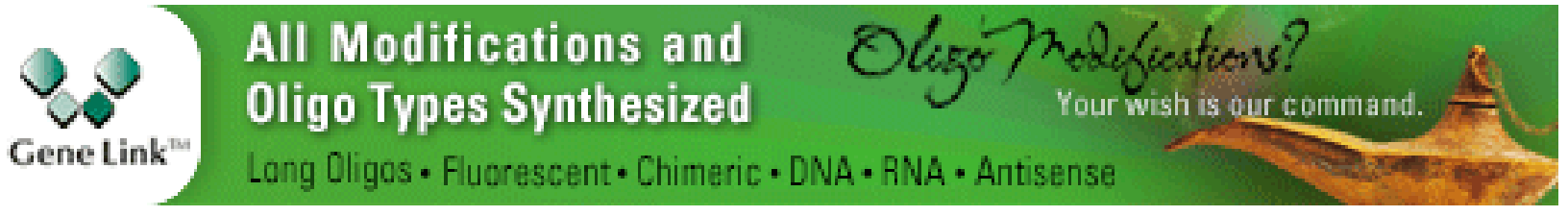

Copyright @ 2010 Cold Spring Harbor Laboratory Press; all rights reserved 